\title{
一种对空红外弱小目标检测跟踪方法研究
}

\author{
王曙光，石胜斌，胡春生 \\ (陆军炮兵防空兵学院, 安徽 合肥 230031)
}

\begin{abstract}
摘要: 为了在对空中小目标打击过程中实现对目标的准确检测与跟踪, 针对空中红外弱小目标信噪比 低、像素点少等特点, 本文基于红外视频图像, 采用高斯滤波以及 Top-Hat 算子对图像进行预处理; 利用边缘检测算法对图像中的目标进行检测与定位; 根据检测得到的目标初始位置，通过核化相关滤 波跟踪算法对目标持续跟踪; 最后对跟踪效果做了定量评估。实验结果显示, 跟踪最大视场角度误差 不超过 $0.0062^{\circ}$, 运行速度平均每帧可达 25.3 帧/s, 该方法能够有效地对空中红外弱小目标进行自动 检测跟踪。
\end{abstract}

关键词: 空中小目标; 红外视频; 高斯滤波; Top-Hat 算子; 边缘检测; 核化相关滤波 (KCF)

中图分类号：TP751 文献标识码：A 文章编号：1001-8891(2020)04-0356-05

\section{Detection and Tracking Method for Infrared Dim Small Targets in Air}

\author{
WANG Shuguang, SHI Shengbin, HU Chunsheng
}

(Army Artillery and Air Defense Academy, Hefei 230031, China)

\begin{abstract}
To detect and track targets accurately when attacking small targets, while attempting to achieve a low signal-to-noise ratio and few pixels of small and dim targets in the air, images are preprocessed based on infrared video images using Gauss filtering and the top-hat operator in this study. The edge detection algorithm is used to detect and locate the target in the image. The initial position of the target is continuously tracked by the kernel correlation filter tracking algorithm. Finally, the tracking effect is quantitatively evaluated. Experimental results show that the tracking error of the maximum field of view angle does not exceed $0.0062^{\circ}$, and that the average running speed can reach $25.3 \mathrm{fps} / \mathrm{s}$. This method can effectively detect and track small and dim infrared targets in the air.
\end{abstract}

Key words: small aerial targets, infrared video, Gauss filtering, top-hat, edge detection, kernelized correlation filter(KCF)

\section{0 引言}

现代战争中, 以小型无人机、电视侦察弹等为代 表的战场侦查设备层出不穷, 对地面部队和武器装备 的生存能力形成较大威胁, 为此, 有效发现、跟踪和 打击该类目标成为必然的战场军事需求。其中目标检 测与跟踪技术作为关键技术, 其性能的优劣直接决定 了打击效果的好坏。常用的获取图像方式有毫米波成 像、可见光成像以及红外成像 3 种。相比较而言, 由 于待探测的目标通常较小，基于毫米波成像效果较 差, 进而会导致跟踪误差较大; 基于可见光成像时, 如果天空中有云, 则目标极有可能与背景混淆, 为目
标的检测与跟踪带来困难; 而基于红外图像时, 目标 在以天空为背景的视野中较为突出, 易于检测, 且背 景较为单一, 干扰较少, 有利于对目标的准确跟踪 ${ }^{[1]}$ 。 综上所述, 宜利用空中小目标的红外视频图像进行检 测与跟踪。

本文利用小型降落伞目标的红外视频, 首先通过 去除噪声、背景抑制、目标增强 3 个步骤对图像进行 预处理, 以提高目标与背景的对比度; 通过轮廓边界 检测得到目标的位置信息; 最后将检测结果传递给跟 踪器, 利用检测得到的目标位置信息通过核化相关滤 波算法, 对目标进行跟踪定位。该方法的流程如图 1 所示。 


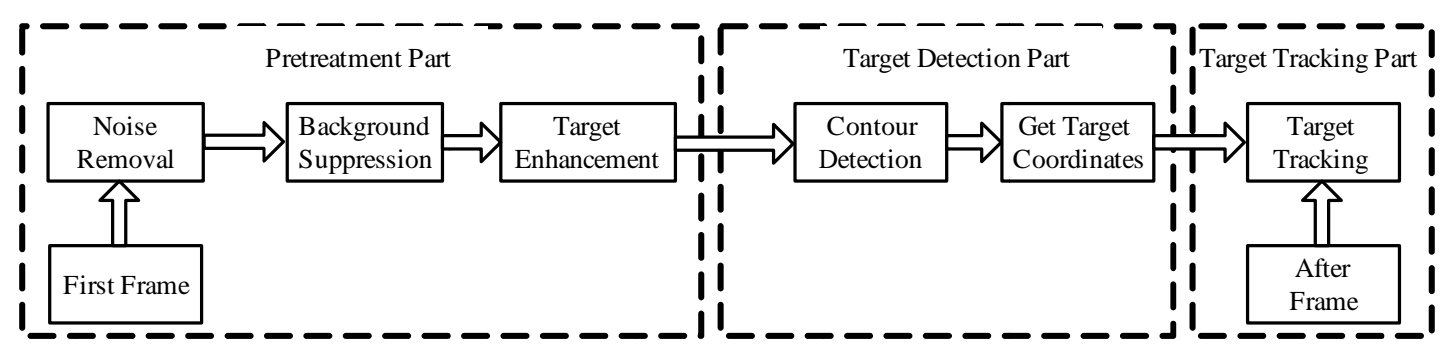

图 1 检测跟踪算法流程图

Fig.1 Flow chart of detection and tracking algorithm

\section{1 红外目标检测}

\section{1 图像预处理}

红外图像预处理的目的是提高图像的信噪比, 通 过减少杂波干扰、抑制背景噪声、增强目标显示效果, 进而提高检测器的检测性能。

\subsection{1 去除噪声}

常用的图像滤波算法包括高斯滤波、最大均值 滤波、中值滤波等, 考虑到红外小目标具有尺寸小、 像素有限、灰度变化不稳定以及背景分布不均匀等 特点 ${ }^{[2]}$, 同时应兼顾算法的运算速度, 故采用高斯滤 波法对输入图像进行去噪处理, 结果如图 2 所示。

\subsection{2 背景抑制}

背景抑制目的是抑制背景杂波和点噪声的干扰, 同时有效地保留目标信息。本文采用形态学 Top-Hat 算子 ${ }^{[3]}$ 对输入图像进行背景抑制处理。

数学形态学运算包括两个基本运算: 膨胀和腐蚀。 设 $f(x, y)$ 是原始灰度图像, $B(p, q)$ 为结构算子。则结构 算子对原始灰度图像的膨胀 $(f \oplus B)$ 和腐蚀运算 $(f \oplus B)$ 的 定义分别为:

$$
\begin{array}{r}
(f \oplus B)(x, y)=\max _{p, q}[f(x-p, y-q)+B(p, q)] \\
(f \oplus B)(x, y)=\min _{p, q}[f(x+p, y+q)-B(p, q)]
\end{array}
$$

基于膨胀和腐蚀运算, 形态学开运算 $(f \vee B)$ 和闭运 算 $(f \wedge B)$ 定义为:

$$
\begin{aligned}
& (f \vee B)(x, y)=[f \otimes B(x, y)] \oplus B(p, q) \\
& (f \wedge B)(x, y)=[f \oplus B(x, y)] \otimes B(p, q)
\end{aligned}
$$

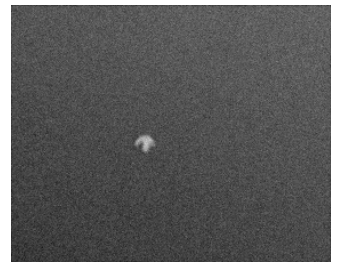

(a) 原图

(a) Original image

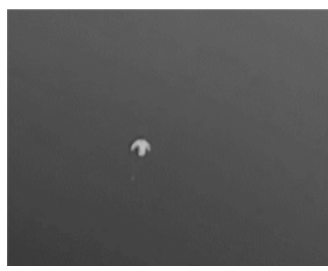

(b) 高斯滤波

(b) Gaussian filtering
Top-Hat 算子定义为:

$$
M_{\text {TopHat }}=f(x, y)-(f \vee B)(x, y)
$$

由于(3)式中开运算的作用为平滑图像中的亮小 区域。公式(5)表明, 形态学 Top-Hat 算子是从原图中 减去开运算后的图, 虽然该方法会使得目标被一定程 度的削弱, 但整个背景的灰度值被显著降低。由图 3 可知, 将去除噪声后的图像与 Top-Hat 算子进行运算, 达到了较好的背景抑制效果, 目标与背景的对比度更 加显著。

\subsection{3 目标增强}

目标增强算法是通过一定方法提升红外图像中 的目标部分的像素值, 增加与背景之间的对比度, 进 而可以更容易地检测出红外图像中的弱小目标 ${ }^{[4]}$ 。我 们通过设定像素阈值（本实验设为 10）的方法, 将图 像中大于该值的像素值等比放大，使得在背景抑制过 程中被削弱目标得到显著增强。实验结果如图 2 所示。

为了更直观地判断预处理效果, 我们以图片的长 和宽为横坐标 $X$ 和纵坐标 $Y$, 像素值为垂直坐标 $Z$, 建立三维图像模型如图 3 所示。

由图可知, 图像经预处理后, 目标与背景的对比 度得到显著增强, 更有利于目标的检测与定位。

\section{2 目标轮廓检测}

在对输入图像进行预处理后, 图像中目标的边缘 像素变化较为明显, 故可以先计算图像中各像素点的 梯度强度 $G$ 及方向 $\theta$ :

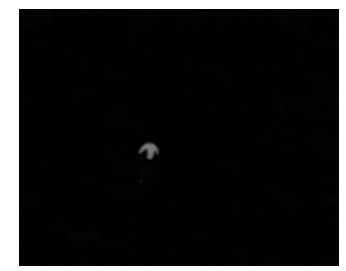

(c) 背景抑制

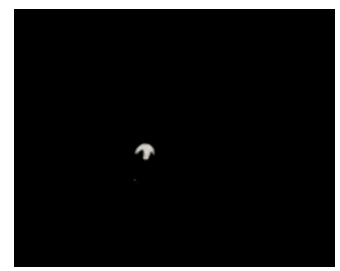

(d) 目标增强
图 2 红外图像预处理效果对比

Fig.2 Comparison of infrared image preprocessing effect 


$$
\begin{gathered}
G=\sqrt{G_{x}{ }^{2}+G_{y}{ }^{2}} \\
\theta=\arctan \left(G_{y} / G_{x}\right)
\end{gathered}
$$

式中: $G_{x} 、 G_{y}$ 分别为像素点在 $x 、 y$ 方向上的梯度分量。

通过非极大值抑制消除杂散效应; 通过双阈值检 测实际边缘位置 ${ }^{[5]}$; 将变化较为明显的像素储存为向量 形式, 最后得到这些向量的最小外接正矩形, 获取目 标的坐标信息并传递给跟踪器。检测结果如图 4 所示。 其中前两个量为目标外接矩形框的左上角坐标值 $(x, y)$, 后两个量为矩形框的宽度和高度 $(w, h)$ 。该参数 用于跟踪器的初始化。

\section{2 核化相关滤波跟踪算法}

核化相关滤波（kernelized correlation filter, KCF) 算法 ${ }^{[6]}$ 借鉴了信号处理中相关滤波的基本原理, 通过

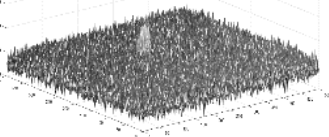

(a) 原图

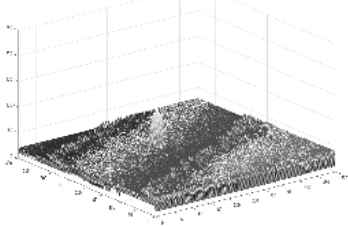

(b) 高斯滤波

(a) Original image

(b) Gaussian filtering

图 3 三维图像模型对比

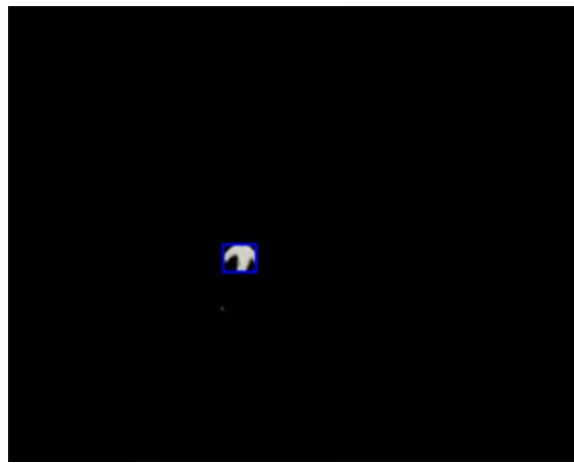

(a) 边缘检测外接矩形

(a) Edge detection circumscribed rectangle

图 4 检测结果
寻找最大响应值的方式来确定目标位置。该算法利用 循环矩阵在目标区域进行循环位移构造大量样本, 解 决了目标跟踪过程中训练样本过少的问题，同时利用 循环矩阵对角化的性质使得计算大大简化。将线性空 间的脊回归函数通过高斯核函数映射到非线性空间, 并同样可以利用循环矩阵的性质简化计算，因此该算 法具有极高的跟踪速度以及较好的跟踪精度。其基本 流程如图 5 所示。

算法的核心问题可以表示为:

$$
f(z)=w^{\mathrm{T}} z
$$

式中: $w$ 是列向量表示权重系数; $z$ 表示样本。 利用岭回归的方法, 其优化函数可由最小二乘以及正 则项组成:

$$
\min _{w} \sum_{i}\left[f\left(x_{i}\right)-y_{i}\right]^{2}+\lambda\|w\|^{2}
$$

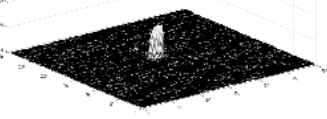

(c) 背景抑制

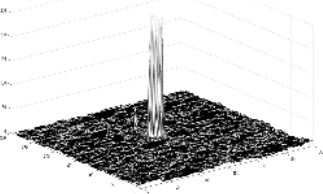

(d) 目标增强 (c) Background suppression

(d) Target enhancement

Fig.3 Comparison of 3D image models

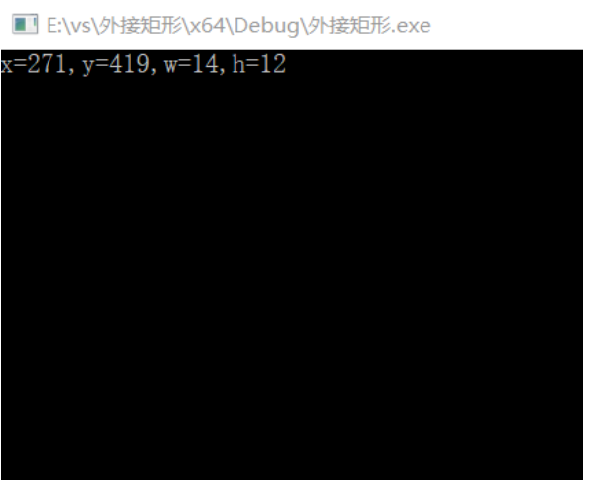

(b) 目标坐标返回值

(b) Target coordinate return value

Fig.4 Detecting results

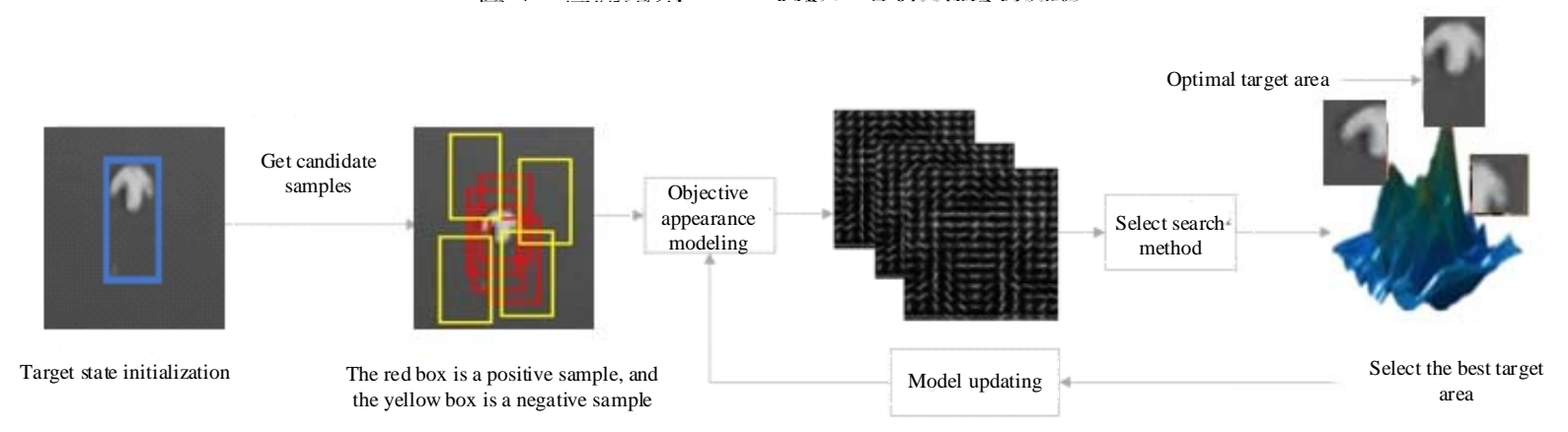

图 $5 \mathrm{KCF}$ 跟踪算法流程

Fig.5 KCF tracking algorithm flow 
式中: $\lambda$ 用于控制系统的结构复杂性; $x$ 为样本行向量; $y$ 为目标函数。若直接求解可得:

$$
w=\left(X^{\mathrm{T}} X+\lambda I\right)^{-1} X^{\mathrm{T}} y
$$

由于共轭以及求逆的计算量较大, 故引入核函数 将问题简化为:

$$
\alpha=(K+\lambda I)^{-1} y
$$

式中: $\alpha$ 为引入核函数后 $w$ 对应解, 利用循环矩阵有 关性质, 将训练过程进一步简化为:

$$
\hat{\alpha}=\frac{\hat{y}}{\hat{k}^{x X}+\lambda}
$$

式中: ^表示复域的计算, 由此即可用此滤波器来对输 入图像进行相关性检测得到跟踪结果, 用公式表示为:

$$
\hat{f}(z)=\hat{k}^{x z} \odot \hat{\alpha}
$$

则响应值 $\hat{f}(z)$ 最大时对应图像中的位置即为目 标位置。目标跟踪性能的实验结果由图 6 可知, 在给 定目标初始位置坐标后, 该算法根据目标特征, 从每 帧图像中获取目标位置, 实现了对目标的准确跟踪。 本方法中目标的检测与跟踪无需手动标注, 整个过程 无需人为控制, 具有较高的自主性与可靠性。

\section{3 实验结果与分析}

为了对跟踪算法性能进行定量测试, 我们利用图 像标注软件得到测试视频每一帧目标位置的标准值, 通过将跟踪器目标位置预测值与标准值进行对比, 进 而计算中心误差以及重叠率。

\section{1 中心误差}

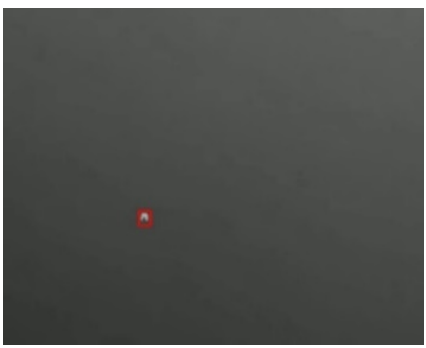

(a) 第 5 帧 (a) 5th frame

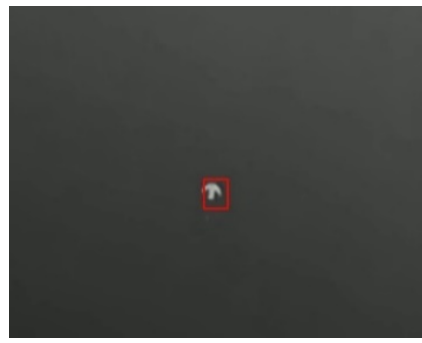

(d) 第 220 帧 (d) 220th frame

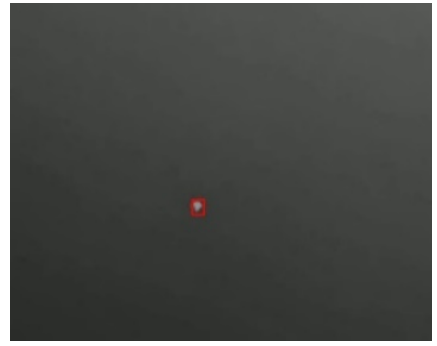

(b) 第 80 帧 $\quad$ (b) 80th frame

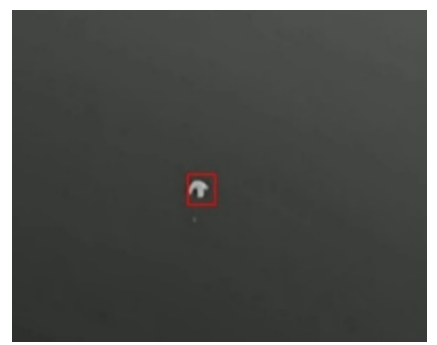

(e) 第 300 帧 (e) 300th frame

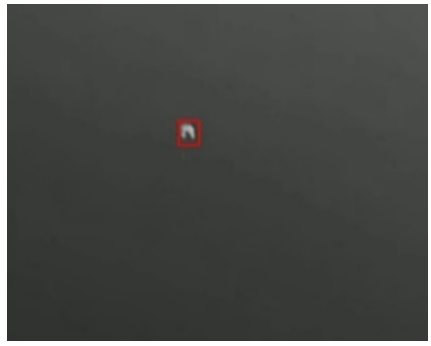

(c) 第 180 帧(c) 180th frame

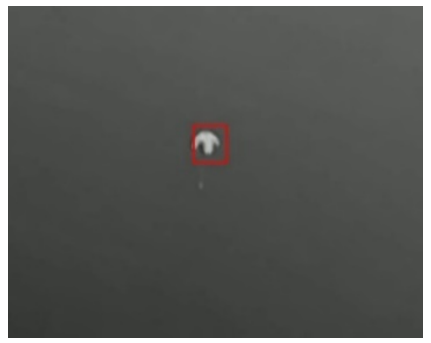

(f) 第 420 帧(f) 420th frame

图 6 KCF 吊伞模型跟踪结果 Fig.6 Tracking results of KCF umbrella model 


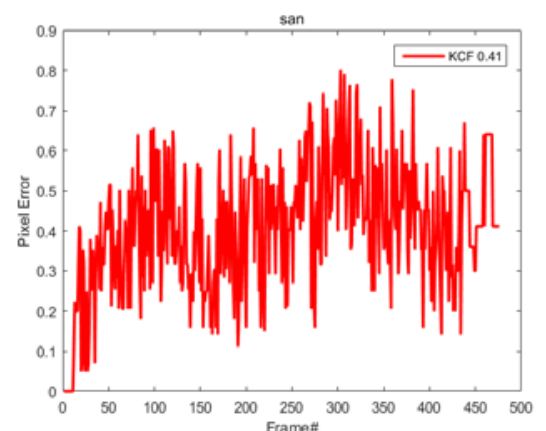

(a) 各帧中心误差 (a) Center error of each frame 图 7 吊伞模型目标跟踪中心误差

由实验结果可知, 算法在对该空中红外小型降落 伞目标的检测及跟踪较为精确, 能够在准确检测到目 标后对目标进行稳定跟踪, 且能够满足实时性要求。

\section{4 总结}

提出了一种人不在回路的对空小目标检测跟踪 方法, 针对红外弱小目标图像像素有限、灰度变化不 稳定以及背景分布不均匀等特点, 通过图像预处理, 保证了边缘轮廓检测效果的可靠性 ${ }^{[8-9]}$; 采用相关滤波 跟踪算法, 保证了该方法的实时性。最后通过实验可 知, 该方法运行速度可达 25.3 帧/s, 且对于该类目标 的视角误差不超过 $0.0062^{\circ}$, 跟踪精度较高, 进一步 印证了本方法在实际应用过程中的可行性, 为有效打 击空中小型目标提供了新的思路。

\section{参考文献:}

[1] 杨丹. 红外弱小运动目标的检测算法研究[D]. 西安: 西安理工大学, 2018.

YANG Dan. Research on Detection Algorithm of Infrared Dim Small Moving Target[D]. Xi'an: Xi'an University of Technology, 2018.

[2] 侯旺, 孙晓亮, 尚洋, 等. 红外弱小目标检测技术研究现状与发展趋 势[J]. 红外技术, 2015, 37(1): 1-10.

HOU Wang, SUN Xiaoliang, SHANG Yang, et al. Research status and development trend of infrared dim small target detection technology[J]. Infrared Technology, 2015, 37(1): 1-10.

[3] 张世锋, 黄心汉, 王敏. 红外背景抑制与小目标检测算法[J]. 中国图 像图形报, 2016, 21(8): 1039-1047.

ZHANG Shifeng, HUANG Xinhan, WANG Min. Infrared background

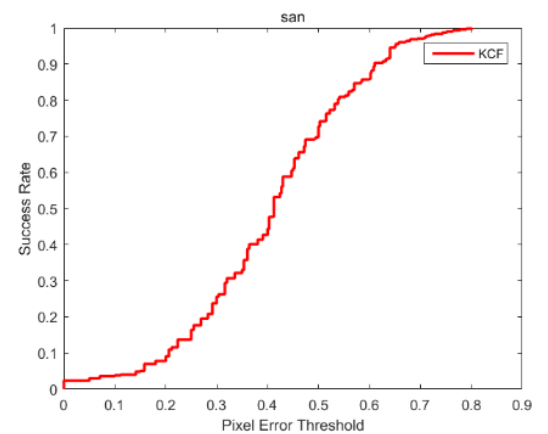

(b) 中心误差率 (b) Center error rate

Fig.7 Target tracking center error of parasol model

suppression and small target detection algorithm[J]. China Image Graphics Daily, 2016, 21(8): 1039-1047.

[4] 赵征. 红外小目标图像预处理与检测算法究[D]. 西安: 西安电子科技 大学, 2014.

ZHAO Zheng. Preprocessing and Detection Algorithm of Infrared Small Target Image[D]. Xi'an: Xi'an University of Electronic Science and Technology, 2014.

[5] 段锁林, 殷聪聪, 李大伟. 改进的自适应 Canny 边缘检测算法 [J]. 计 算机工程与设计, 2018, 39(6): 1645-1652.

DUAN Suolin, YIN Congcong, LI Dawei. Improved adaptive Canny edge detection algorithm[J]. Computer Engineering and Design, 2018, 39(6): 1645-1652.

[6] Henriques J F, Rui C, Martins P, et al. High-speed tracking with Kernelized correlation filters[J]. IEEE TPAMI, 2015, 37(3): 583-596.

[7] WU Y, Lim J, YANG M H. Online object tracking: a benchmark[C]/IEEE Conference on Computer Vision and Pattern Recognition, 2013: 23-28.

[8] 危水根, 王程伟, 张聪炫, 等. 多信息融合的红外弱小目标检测 [J]. 红 外技术, 2019, 41(9): 857-865.

WEI Shuigen, WANG Chengwei, ZHANG Congxuan, et al. Infrared dim target detection based on multi-information fusion[J]. Infrared Technology, 2019, 41(9): 857-865.

[9] 杨慧, 张宝辉, 沙涛, 等. 地空背景下红外弱小运动目标检测 [J]. 红外 技术, 2018, 40(5): 462-467.

YANG Hui, ZHANG Baohui, SHA Tao, et al. Detection of small infrared moving targets under ground-sky background[J]. Infrared Technology, 2018, 40(5): 462-467 